Open Access

\title{
A randomized phase II/III study of adverse events between sequential (SEQ) versus simultaneous integrated boost (SIB) intensity modulated radiation therapy (IMRT) in nasopharyngeal carcinoma; preliminary result on acute adverse events
}

Anussara P. Songthong, Danita Kannarunimit, Chakkapong Chakkabat and Chawalit Lertbutsayanukul ${ }^{*}$

\begin{abstract}
Background: To investigate acute and late toxicities comparing sequential (SEQ-IMRT) versus simultaneous integrated boost intensity modulated radiotherapy (SIB-IMRT) in nasopharyngeal carcinoma (NPC) patients.

Methods: Newly diagnosed stage I-IVB NPC patients were randomized to receive SEQ-IMRT or SIB-IMRT, with or without chemotherapy. SEQ-IMRT consisted of two sequential radiation treatment plans: 2 Gy $\times 25$ fractions to low-risk planning target volume (PTV-LR) followed by $2 \mathrm{~Gy} \times 10$ fractions to high-risk planning target volume (PTV-HR). In contrast, SIB-IMRT consisted of only one treatment plan: 2.12Gy and 1.7Gy $\times 33$ fractions to PTV-HR and PTV-LR, respectively. Toxicities were evaluated according to CTCAE version 4.0.

Results: Between October 2010 and November 2013, 122 eligible patients were randomized between SEQ-IMRT (54 patients) and SIB-IMRT (68 patients). With median follow-up time of 16.8 months, there was no significant difference in toxicities between the two IMRT techniques. During chemoradiation, the most common grade 3-5 acute toxicities were mucositis ( $15.4 \%$ vs $13.6 \%$, SEQ vs SIB, $p=0.788$ ) followed by dysphagia (9.6\% vs $9.1 \%, p=1.000$ ) and xerostomia ( $9.6 \%$ vs $7.6 \%, p=0.748$ ). During the adjuvant chemotherapy period, $25.6 \%$ and $32.7 \%$ experienced grade 3 weight loss in SEQ-IMRT and SIB-IMRT $(p=0.459)$. One-year overall survival (OS) and progression-free survival (PFS) were $95.8 \%$ and $95.5 \%$ in SEQ-IMRT and $98 \%$ and $90.2 \%$ in SIB-IMRT, respectively ( $p=0.472$ for OS and 0.069 for PFS).

Conclusion: This randomized, phase II/III trial comparing SIB-IMRT versus SEQ-IMRT in NPC showed no statistically significant difference between both IMRT techniques in terms of acute adverse events. Short-term tumor control and survival outcome were promising.
\end{abstract}

Keywords: Intensity-Modulated Radiotherapy (IMRT), Simultaneous integrated boost, Nasopharyngeal carcinoma

\footnotetext{
*Correspondence: chulacancer@yahoo.com

Division of Radiation Oncology, Department of Radiology, Faculty of

Medicine, Chulalongkorn University, King Chulalongkorn Memorial Hospital,

1873 Rama IV Rd, Pathumwan, Bangkok 10330, Thailand
} 


\section{Background}

Nasopharyngeal carcinoma (NPC) is one of the most common head and neck tumors and has a good prognosis. The worldwide age-standardized incidence rates of NPC are 1.7 and 0.7 per 100,000 in males and females, respectively, whereas this increases to 6.4 and 2.4 in Southeast Asia. In Thailand, the age-standardized incidence rates of NPC are approximately 3.3 and 1.1 per 100,000 in males and females, respectively [1]. Radiotherapy is the standard treatment for NPC, due to its location and radiosensitivity.

In consideration of the proximity of many critical structures to the nasopharynx, including parotid glands, eyes, brain stem and spinal cord, it is challenging to provide an adequate dose to the tumor while sparing those surrounding tissues. An advanced radiotherapy technique, Intensity Modulated Radiation Therapy (IMRT), has been developed with the clear advantages of highly conformal and precise coverage with sharp dose gradients shown by many dosimetric studies; it has been impressively embraced in treatment of head and neck tumors [2-4]. In clinical studies, the use of IMRT can provide better preservation and faster recovery of parotid gland function [5-7].

IMRT may be applied in the same fractionation scheme as in conventional 3D Conformal Radiation Therapy (3DCRT). Initially, the target volume and elective nodal regions are treated in the large field followed by a sequential boost to the gross tumor volume within the smaller field, the so called shrinking field technique or sequential technique (SEQ-IMRT). Subsequently, a new fractionation scheme has been initiated as an accelerated fractionation, known as simultaneous integrated boost IMRT (SIBIMRT), which offers the opportunity to treat both primary and secondary targets simultaneously at different doses, so-called "dose painting" [8]. Compared to sequential treatment, SIB-IMRT uses only one radiation treatment plan for the entire course of treatment.

These two IMRT techniques are widely used in current practice [5-10]. According to dosimetric analysis, the target coverage from SEQ-IMRT and SIB-IMRT is the same, while SIB-IMRT provides superior dose distribution to the elective nodal area and better sparing of parotid glands and inner ear structures. Despite the promising results of SIB-IMRT for NPC, using a high dose per fraction to the target may result in higher dose per fraction to adjacent normal tissue, which can lead to more acute or late toxicity [11]. However, clinical studies comparing SIB-IMRT and sequential IMRT are scarce. Most clinical results of NPC treated by IMRT use the SIB technique [6, 11-18] and rarely compare with the sequential one.

The objectives of this study are to assess acute and late toxicities comparing SIB-IMRT with SEQ-IMRT and to evaluate clinical outcomes including tumor response, pattern of failure and survival of NPC patients treated with both IMRT techniques.

\section{Methods}

\section{Patients and methods}

Patients with newly diagnosed, histopathologic confirmed squamous cell carcinoma of the nasopharynx, stage I-IVB were eligible. Patients had to be at least 18 years of age with a Karnofsky performance status of at least 70 and adequate bone marrow, renal and liver function. The exclusion criteria included previous irradiation for a head and neck tumor or prior chemotherapy within six months, any other experimental therapeutic cancer treatment and any other malignancy except nonmelanoma skin cancer or a carcinoma not of head and neck origin and controlled at least five years. Prophylactic use of amifostine or pilocarpine or concurrent use of amifostine, pilocarpine, diuretics, antidepressant or anticholinergic drugs that affect saliva secretion was prohibited.

All eligible patients received a pretreatment evaluation including complete history and physical examination, endoscopic biopsy, routine laboratory tests for hematologic, renal and hepatic function as well as a dental and nutritional evaluation prior to treatment. Radiological investigations consisted of computed tomography (CT) scan or magnetic resonance imaging (MRI) of the nasopharynx, chest radiography, ultrasound of the upper abdomen and bone scintigraphy. Positron emission tomography (PET) scan was optional. Pathologic confirmation of NPC was performed and re-classified according to WHO subtype [19].

\section{Study design}

This study was a phase II/III randomized controlled trial approved by the institutional review board (IRB no.424/ 53) and patients provided written informed consent prior to the randomization. According to the effects of tumor and nodal burden of the disease on the adverse effects and clinical outcome [16], our patients were stratified by their $\mathrm{T}$ stages (T1-T3 versus $\mathrm{T} 4$ ) and $\mathrm{N}$ stages (N0-N1 versus N2-N3). The patients in each group were then randomized into two arms: the SEQIMRT arm and SIB-IMRT arm, using the stratified block randomization method.

\section{Treatment protocol \\ Radiotherapy}

All patients were immobilized in the supine position with a tailored head-shoulder thermoplastic mask. A CT simulation was performed and transferred to the Eclipse planning system Version 8.6 (Varian Medical System, Palo Alto, CA). If available, MR images were co-registered with CT images. Target volume delineation was performed 
according to the RTOG 0225 protocol. Two planning target volumes (PTVs) were designed: PTV-High Risk (PTVHR) and PTV-Low Risk (PTV-LR). The dose prescriptions were 70 Gy for PTV-HR at 2.12 Gy/fraction and 56 Gy for PTV-LR at $1.7 \mathrm{~Gy} /$ fraction delivered in 33 fractions for SIB-IMRT. For SEQ-IMRT, all PTVs were delivered at $2 \mathrm{~Gy} /$ fraction for 25 fractions with total dose of $50 \mathrm{~Gy}$, followed by a boost of 20 Gy in 10 fractions to PTV-HR. The whole-field IMRT was applied in both techniques. Dosimetric difference, i.e., Biological Equivalent Dose (BED), between the two techniques is shown in Table 1.

Radiation treatment interruptions were allowed if there was any toxicity that affected patient's safety or quality of life. If the treatment break exceeded 14 days, the patient was removed from protocol treatment but was then administered complete treatment at the discretion of the physician and was included in the study in the intention-to-treat analysis.

\section{Chemotherapy}

Cisplatin, $40 \mathrm{mg} / \mathrm{m}^{2}$, was administered weekly concurrently with IMRT to those patients with more than T1 or positive nodal disease for the maximum of seven cycles. Subsequently, one month after completion of chemoradiation, adjuvant chemotherapy was given at four-week interval for three cycles. Each cycle consisted of cisplatin $80 \mathrm{mg} / \mathrm{m}^{2}$ and 5 -FU $1000 \mathrm{mg} / \mathrm{m}^{2} / 24 \mathrm{~h}$ over a $96 \mathrm{~h}$ continuous infusion. In cases of toxicity, chemotherapy dosage modifications for the following cycles were based upon nadir counts and interim nonhematologic toxicities of the preceding cycle.

\section{Treatment monitoring and evaluation}

Toxicity was reassessed at weekly intervals during chemoradiation, before each cycle of adjuvant chemotherapy and as regularly scheduled. Acute and late toxicities (defined as beyond three months after the completion of treatment) were recorded according to the Common Terminology Criteria for Adverse Events (CTCAE)

Table 1 Dosimetric difference between SIB-IMRT and SEQ-IMRT strategies

\begin{tabular}{lll}
\hline Technique & BED (tumor) & BED (late) \\
\hline SIB-IMRT (1-phase) & & \\
PTV-HR $=2.12 \times 33=70 \mathrm{~Gy}$ & $84.8 \mathrm{~Gy}_{10}$ & $119.4 \mathrm{~Gy}_{3}$ \\
PTV-LR $=1.7 \times 33=56 \mathrm{~Gy}$ & $65.6 \mathrm{~Gy}_{10}$ & $87.9 \mathrm{~Gy}_{3}$ \\
SEQ-IMRT $(2$-phase $)$ & & \\
PTV-HR $=(2 \times 25)+(2 \times 10)=70 \mathrm{~Gy}$ & $84.00 \mathrm{~Gy}_{10}$ & $116.7 \mathrm{~Gy}_{3}$ \\
PTV-LR $=2 \times 25=50 \mathrm{~Gy}$ & $60.00 \mathrm{~Gy}_{10}$ & $83.3 \mathrm{~Gy}_{3}$ \\
\hline
\end{tabular}

Biological effective dose (BED) calculation is based on the linear-quadratic model for cell kill with an $\alpha$ / $\beta$ ratio of 10 for tumors and an $\alpha / \beta$ ratio of 3 for late-responding normal tissue

BED Total Dose (Gy) $x(1+$ dose per fraction(Gy)) $(\alpha / \beta)$ version 4.03. CT or MRI of the nasopharynx was done at three months after the completion of chemoradiation to determine tumor response, using Response Evaluation Criteria in Solid Tumors (RECIST) criteria version 1.1. Fiberoptic nasopharyngeal examination was routinely performed 12 weeks after treatment completion.

\section{Statistical analysis}

The primary end points of the treatment were acute and late toxicities. According to RTOG 0225 results in 57 NPC patients treated with SIB-IMRT with or without chemotherapy, severe acute adverse effects occurred in $78 \%$ including $61.8 \%$ grade $3,11.8 \%$ grade 4 and $4.4 \%$ grade 5 [13]. Compared to our data, 73 NPC patients mainly $(91.8 \%)$ treated with SEQ-IMRT with carboplatin-based chemotherapy, severe acute toxicity (grade 3-5) occurred in $16.4 \%$ of patients which was much lower than RTOG data [20]. To detect a $20 \%$ difference between these two techniques with a power of $80 \%$, a p-value of 0.05 and $10 \%$ drop out rate, a total of 218 patients were required. The investigators report here the preliminary results of 122 patients $(56 \%)$ accrued to date in this randomized phase III study. The secondary end points were to assess tumor response, pattern of failure and survival. Progression-free survival (PFS) was defined as the date of treatment initiation to the date of either disease progression or death. Overall survival (OS) was defined as the date of treatment initiation to the date of death, regardless of the cause.

Comparison of toxicity grading between treatment groups was statistically analyzed using Chi-square test. Survival analyses were computed using the KaplanMeier method and log-rank test. Statistical Packages for Social Sciences (SPSS) software version 17.0 was used for statistical analysis.

\section{Results}

Between October 2010 and November 2013, a total of 130 patients of biopsy-proven NPC stage I-IVB was enrolled in this study. One hundred and twenty-two patients who completed chemoradiation and had followed up for more than 3 months were analyzed. Patient and disease characteristics are described in Table 2. Patients in SIB-IMRT had slightly more advanced disease; however, there was no statistically significant difference. Approximately $89 \%$ of the patients had non-keratinizing undifferentiated squamous cell carcinoma. Median followup time was 16.8 months.

Dosimetric data were summarized in Table 3. There was a statistically significant difference in average PTVLR dose and maximal dose to spinal cord and eyes as well as median dose to one parotid gland and cochlear between the two treatment arms. The physical PTV-LR dose difference resulted from the different prescription 
Table 2 Patient demographic and baseline characteristics $(N=122)$

\begin{tabular}{|c|c|c|c|c|}
\hline Characteristics & All $(N=122)$ & SEQ $(N=54)$ & $\mathrm{SIB}(\mathrm{N}=68)$ & $p$-value \\
\hline Age, years & & & & 0.680 \\
\hline Mean \pm SD & $49.39 \pm 10.30$ & $49.7 \pm 10.63$ & $48.96 \pm 9.96$ & \\
\hline Sex & & & & 0.563 \\
\hline Male & 95 (77.9 \%) & 39 (72.2 \%) & $56(82.4 \%)$ & \\
\hline Female & $27(22.1 \%)$ & $15(27.8 \%)$ & $12(17.6 \%)$ & \\
\hline Performance status & & & & - \\
\hline $90-100$ & $122(100 \%)$ & $54(100 \%)$ & $68(100 \%)$ & \\
\hline WHO classification & & & & 0.983 \\
\hline Type I (Keratinizing SCCA) & $2(1.6 \%)$ & $1(1.9 \%)$ & $1(1.5 \%)$ & \\
\hline Type IIA (NK, diff. SCCA) & $11(9.0 \%)$ & $5(9.2 \%)$ & $6(8.8 \%)$ & \\
\hline Type IIB (NK,undiff. SCCA) & $109(89.4 \%)$ & 48 (88.9 \%) & 61 (89.7\%) & \\
\hline T stage & & & & 0.741 \\
\hline 1 & 35 (28.7\%) & 17 (31.5\%) & $18(26.5 \%)$ & \\
\hline 2 & $50(41.0 \%)$ & $22(40.7 \%)$ & 28 (41.2\%) & \\
\hline 3 & $24(19.7 \%)$ & $11(20.4 \%)$ & $13(19.1 \%)$ & \\
\hline 4 & $13(10.6 \%)$ & $4(7.4 \%)$ & $9(13.2 \%)$ & \\
\hline N stage & & & & 0.910 \\
\hline 0 & $5(4.1 \%)$ & $2(3.7 \%)$ & $3(4.4 \%)$ & \\
\hline 1 & $22(18.0 \%)$ & $9(16.6 \%)$ & 13 (19.1\%) & \\
\hline 2 & 77 (63.2 \%) & $36(66.7 \%)$ & $41(60.3 \%)$ & \\
\hline 3 & $18(13.8 \%)$ & $7(13.0 \%)$ & $11(16.2 \%)$ & \\
\hline AJCC Stage grouping & & & & 0.259 \\
\hline$\|$ & $18(14.8 \%)$ & $8(14.8 \%)$ & 10 (14.7\%) & \\
\hline III & 75 (61.4\%) & 37 (68.5\%) & 38 (55.9\%) & \\
\hline IVA & $11(9.0 \%)$ & $2(3.7 \%)$ & $9(13.2 \%)$ & \\
\hline IVB & 18 (14.8\%) & $7(13.0 \%)$ & 11 (16.2\%) & \\
\hline Mean PTV volume, cc & & & & 0.432 \\
\hline $\mathrm{PTV}-\mathrm{HR} \pm \mathrm{SD}$ & $366.29 \pm 122.07$ & $354.76 \pm 105.61$ & $376.68 \pm 135.70$ & \\
\hline$P T V-L R \pm S D$ & $813.7 \pm 150.52$ & $802.98 \pm 146.84$ & $823.37 \pm 154.98$ & 0.558 \\
\hline RT duration, days & & & & 0.002 \\
\hline Mean \pm SD & $49.36 \pm 3.72$ & $50.54 \pm 3.87$ & $48.43 \pm 3.33$ & \\
\hline
\end{tabular}

AJCC American Joint Committee of Cancer, SEQ Sequential IMRT arm, SIB Simultaneous integrated boost IMRT arm, PTV Planning target volume, HR High risk region, $L R$ low-risk region

doses between the two techniques with regards to BED as shown in Table 1.

\section{Treatment compliance}

The CONSORT diagram of treatment is presented in Fig. 1. Treatment interruption occurred in two patients from each arm due to grade 3 neutropenia and a flooding disaster in Thailand; the total radiation durations of these patients were 61 and 63 days, respectively. Of the 122 patients, 25 patients $(20.5 \%)$ completed seven cycles of concurrent weekly cisplatin and 115 patients (94.3\%) received at least four cycles; 52 patients
(96.3\%) and 63 patients (92.6\%) in SEQ-IMRT and SIB-IMRT, respectively. One patient in SEQ-IMRT received radiation alone due to advanced age and died before chemotherapy. Eighty patients (65.6\%) completed all three cycles of adjuvant chemotherapy; 32 patients (59.3\%) and 48 patients (70.6\%) in SEQ-IMRT and SIB-IMRT, respectively. The reasons contributing to discontinuation during treatment included treatmentrelated toxicities; for example, prolonged grade 2 neutropenia, severe nausea and anorexia. One patient in SIB-IMRT was unable to receive adjuvant chemotherapy due to prolonged neutropenia. 
Table 3 Dosimetric comparison of two IMRT techniques

\begin{tabular}{|c|c|c|c|}
\hline \multirow[t]{2}{*}{ Target / Organ at risk } & \multicolumn{2}{|c|}{ Average dose (Mean \pm SD, Gy) } & \multirow[b]{2}{*}{$p$-value } \\
\hline & SEQ & $\mathrm{SIB}$ & \\
\hline PTV-HR dose (D95\%) & $69.80 \pm 2.66$ & $69.47 \pm 2.00$ & 0.452 \\
\hline PTV-LR dose (D95\%) & $52.21 \pm 3.22$ & $56.71 \pm 2.07$ & 0.000 \\
\hline Maximal spinal cord dose (D1cc) & $40.65 \pm 4.34$ & $42.09 \pm 2.23$ & 0.030 \\
\hline Maximal brain stem dose (D1cc) & $48.84 \pm 6.02$ & $50.13 \pm 4.44$ & 0.193 \\
\hline Median dose of one parotid gland (D50\%) & $24.44 \pm 8.69$ & $21.91 \pm 4.04$ & 0.035 \\
\hline Maximal optic nerve dose & $30.06 \pm 13.99$ & $34.26 \pm 16.58$ & 0.132 \\
\hline Median cochlear dose (D50\%) & $47.26 \pm 8.52$ & $44.01 \pm 7.84$ & 0.032 \\
\hline Maximal eye dose & $19.40 \pm 7.34$ & $23.05 \pm 9.10$ & 0.016 \\
\hline Maximal lens dose & $6.44 \pm 1.78$ & $7.91 \pm 1.07$ & 0.149 \\
\hline Maximal mandible dose & $71.27 \pm 5.22$ & $71.02 \pm 5.74$ & 0.802 \\
\hline Median oral cavity dose (D50\%) & $37.28 \pm 4.21$ & $36.40 \pm 4.30$ & 0.256 \\
\hline Median vocal cord dose (D50\%) & $39.55 \pm 6.16$ & $40.97 \pm 4.85$ & 0.667 \\
\hline
\end{tabular}

${ }^{a}$ The smallest dose between bilateral parotid glands

\section{Acute and late toxicity}

We classified toxicities into acute and late toxicities by three-month periods after completion of the adjuvant chemotherapy. All patients experienced some degrees of acute toxicities, most of which were mild severity (grade 1-2). Severe toxicities (grade 3-5) during concurrent chemoradiation occurred in 18 patients (14.7\%), 9 patients $(16.7 \%)$ in SEQ-IMRT and 9 patients $(13.2 \%)$ in SIB-IMRT ( $p$-value $=0.788)$, most of which was oral mucositis. One treatment-related death was suspected in a 79 year-old man in SEQ-IMRT due to severe oral mucositis and electrolyte imbalance.

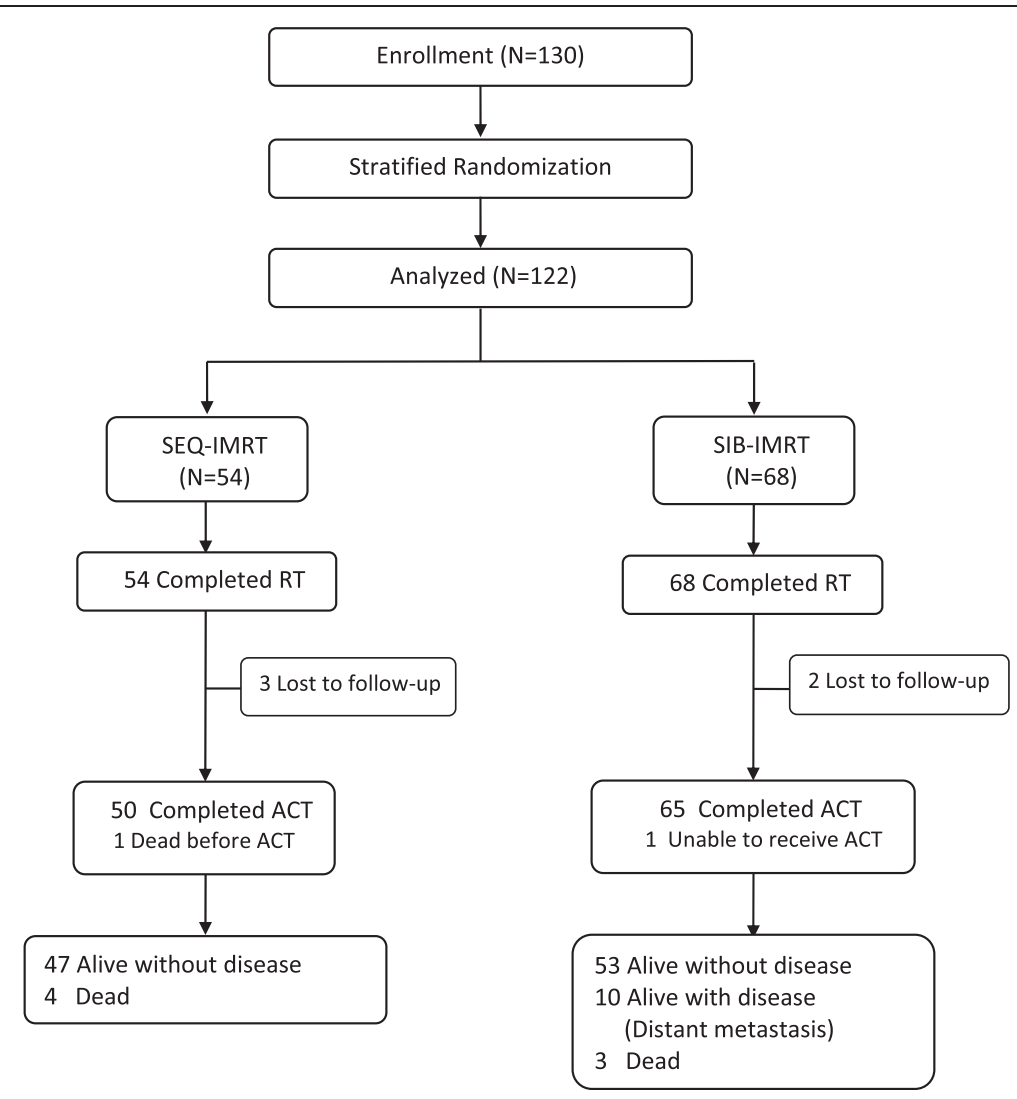

Fig. 1 CONSORT diagram showing the flow of participants. Abbreviation: RT = Radiotherapy, ACT = Adjuvant Chemotherapy 
During the adjuvant chemotherapy period, the incidence of acute grade 3 weight loss (more than $20 \%$ from baseline) was 10 patients (20\%) in SEQ-IMRT and 18 patients $(27.3 \%)$ in SIB-IMRT ( $p$-value $=0.459)$. However, there was no significant difference in severity of acute toxicity during both treatment periods between the two techniques, as described in Table 4.

During follow-up after the completion of adjuvant treatment, the incidence of grade 3 weight loss was 8 patients (26.7 \%) in SEQ-IMRT and 15 patients (34.9 \%) in SIBIMRT, $p$-value $=0.457$. None of patients experienced grade 3-4 gastrointestinal, dermatologic and hematologic toxicities during follow-up. Neither renal function impairment nor treatment-related death was found. In the threemonth period, rates of grade 1 and grade 2 xerostomia were $24.3 \%$ and $9.5 \%$, respectively. No patient experienced more than grade 2 xerostomia. Overall, there was no significant difference in severity of late toxicity between SEQ-IMRT and SIB-IMRT.

\section{Tumor response}

Of the 122 patients, 115 patients could be evaluated for response by performing a CT scan of the nasopharynx at three months after completion of radiation. Five patients were lost to follow up and 2 patients died before evaluation. According to RECIST criteria version 1.1, 41 patients $(83.7 \%)$ in SEQ-IMRT and 56 patients $(84.8 \%)$ in SIB-IMRT achieved complete remission. Eight patients $(16.3 \%)$ and 10 patients (15.2 \%) had partial response in SEQ-IMRT and SIB-IMRT, respectively. One patient had residual primary disease and received additional boost with photon of $3 \mathrm{~Gy} /$ fraction for 10 fractions at 5 months after complete chemoradiation. Seventeen patients had residual cervical lymph nodes and received additional boost with 6-9 MeV electron $3 \mathrm{~Gy} /$ fraction for 5 fractions. Three of them further received salvage neck dissection but all were pathologically negative.

Table 4 Severe (grade 3-5) acute toxicity during concurrent chemoradiation and adjuvant chemotherapy

\begin{tabular}{|c|c|c|c|}
\hline & SEQ & $\mathrm{SIB}$ & $p$-value \\
\hline \multicolumn{4}{|c|}{ Severe acute toxicity during concurrent chemoradiation } \\
\hline Weight loss & $5.9 \%$ & $6.2 \%$ & $1.000^{*}$ \\
\hline Oral mucositis & $15.4 \%$ & $13.6 \%$ & 0.788 \\
\hline Dysphagia & $9.6 \%$ & $9.1 \%$ & $1.000^{*}$ \\
\hline Xerostomia & $9.6 \%$ & $7.6 \%$ & $0.748^{*}$ \\
\hline Dermatitis & $7.7 \%$ & $9.1 \%$ & $1.000^{*}$ \\
\hline \multicolumn{4}{|c|}{ Severe acute toxicity during adjuvant chemotherapy } \\
\hline Weight loss & $20 \%$ & $27.3 \%$ & 0.459 \\
\hline Dysphagia & $2 \%$ & $1.5 \%$ & $1.000^{*}$ \\
\hline
\end{tabular}

*Fischer's exact test was used due to low expected count data

\section{Pattern of failure}

Locoregional recurrence was found in only one patient $(1.5 \%)$ in SIB-IMRT. None in SEQ-IMRT developed distant metastasis while this occurred in nine patients $(13.6 \%)$ in SIB-IMRT ( $p$-value $=0.005)$. Seven patients (5.7\%) died.

\section{Survival outcome}

Survival analysis was performed using Kaplan-Meier survival analysis, as shown in Figs 2 and 3. For overall and in both treatment groups, the median survival was not reached at the time of analysis. One-year overall survival (OS) and progression-free survival (PFS) were $95.8 \%$ and $95.5 \%$ in SEQ-IMRT and $98 \%$ and $90.2 \%$ in SIBIMRT ( $p$-value $=0.472$ for OS and 0.069 for PFS), respectively.

\section{Discussion}

Radiotherapy with or without chemotherapy has been the standard treatment for nasopharyngeal carcinoma [21]. Several studies have shown the popularity and benefits of the advanced radiation technique, IMRT, which can achieve dose escalation to the tumor and also reduce the dose to the surrounding normal tissue [2-4]. IMRT may be applied in either sequential (SEQ) technique or simultaneous integrated boost (SIB) technique. SIBIMRT needs only a single treatment plan, which is more convenient and more efficient than SEQ-IMRT. From a socioeconomic prospective, fewer treatment fractions (i.e., 33 fractions in SIB-IMRT as compared to 35 fractions in SEQ-IMRT) also lead to time and cost savings as well as reducing the workload of heath care providers. Moreover, from the radiobiological point of view, reduction in overall treatment time is assumed to reduce the risk of tumor clonogens regrowth during the late phase of radiation treatment and probably to improve tumor control probability [22]. In our study, the BED to PTVHR (PTV-70) was slightly higher in SIB-IMRT than SEQ-IMRT, 84.8 $\mathrm{Gy}_{10}$ and 84.0 $\mathrm{Gy}_{10}$, respectively. This higher fraction size might have an effect on cellular death and regeneration of acutely responding normal tissue surrounding target volumes. Denham reported an earlier time of adverse effects during accelerated RT than conventional RT [23].

Chen [11] and Nesrin [24] performed dosimetric studies comparing the target volume coverage and normal tissue sparing of SIB-IMRT versus SEQ-IMRT for nasopharyngeal carcinoma. Both of them found that target coverage was the same for both techniques while SIBIMRT provided superior dose distribution to the elective nodal area and better sparing of the parotid glands and inner ear structures. This is the same direction in our study which showed that the median dose to one parotid gland and cochlear in SEQ-IMRT $(24.44 \pm 8.69$ Gy and 


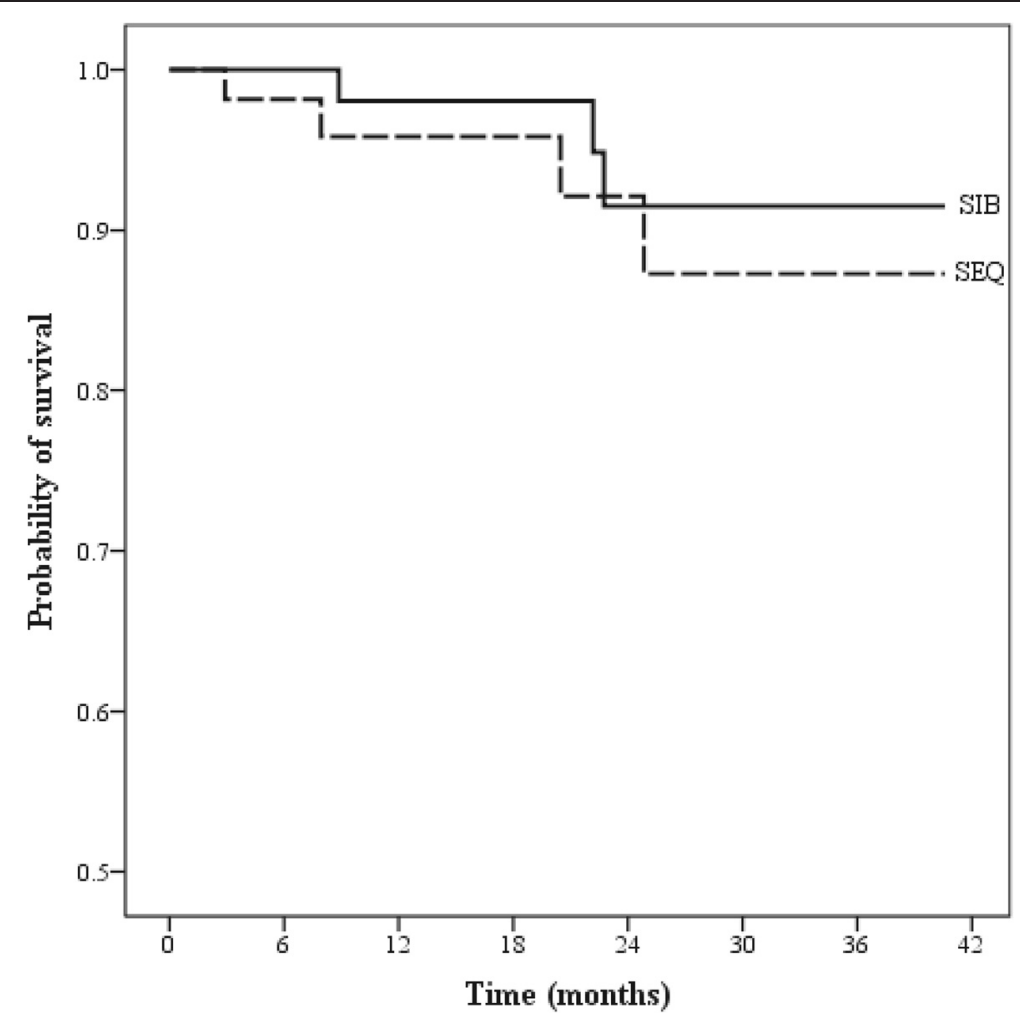

Fig. 2 Overall survival between two IMRT techniques

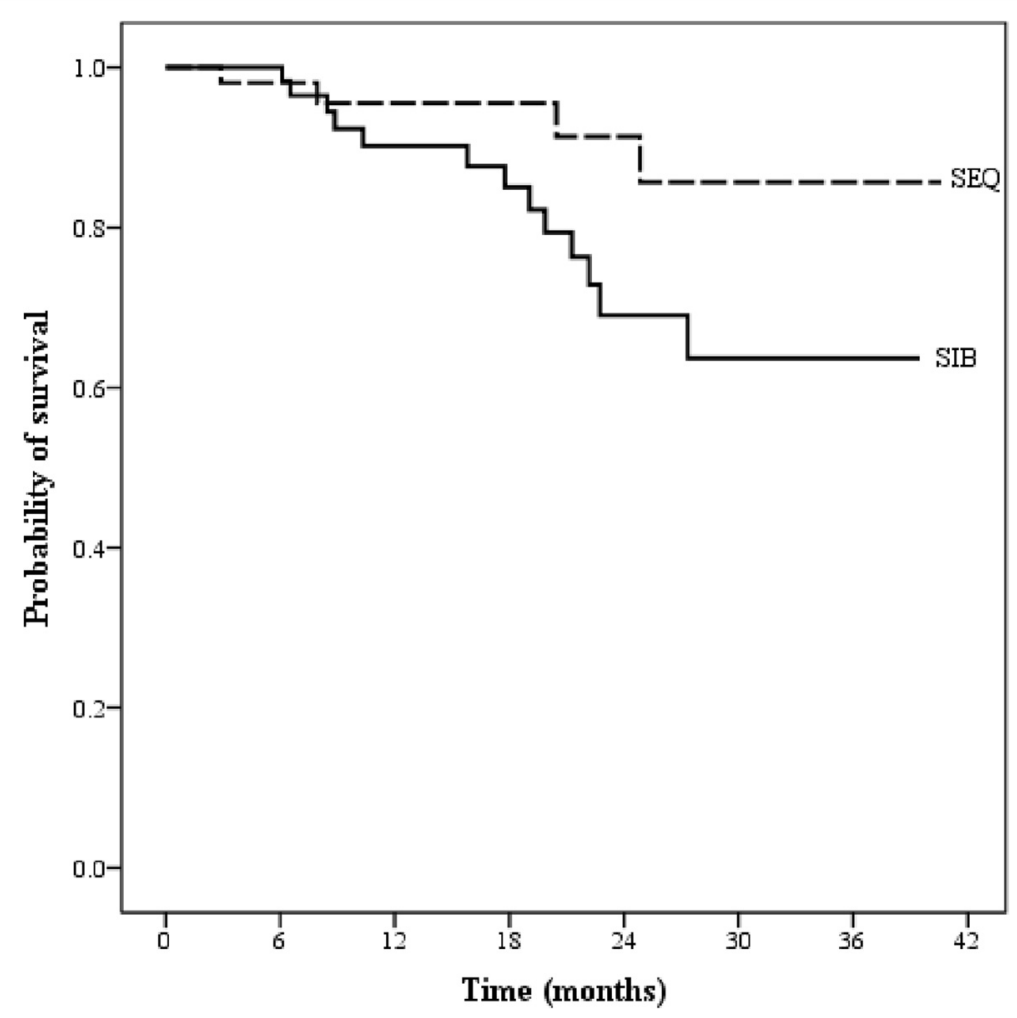

Fig. 3 Progression-free survival between two IMRT techniques 
47.26 \pm 8.52 Gy) were higher than in SIB-IMRT (21.91 \pm 4.04 Gy and $44.01 \pm 7.84$ Gy) ( $p=0.035$ for parotid and $p=0.032$ for cochlear). Additionally, the median oral cavity dose was higher in SEQ-IMRT despite no significant difference $(p=0.256)$. The possible explanation was the sum of two treatment plans in SEQ-IMRT resulted in expansion of low-dose area. However, it was also noted that critical organs close to the gross target volume, brain stem and spinal cord as well as optic nerves and eyes especially in T4 cases, might receive higher doses when applying SIB-IMRT due to higher prescription dose to PTV-LR in SIB-IMRT. However, the dose to these organs was within acceptable constraint. We found that the small difference of parotid dose between SIB and SEQ-IMRT did not translate to different clinical outcome.

Most clinical studies and a review of SIB-IMRT in NPC demonstrated excellent clinical outcomes in terms of tumor control and acceptable acute and late toxicities [12-18, 25]. However, clinical studies comparing SIBIMRT and SEQ-IMRT are scarce. In our institute, SEQIMRT and SIB-IMRT have been used in the treatment of nasopharyngeal cancer since 2005, according to physicians' preference. Lertbutsayanukul initially used SEQIMRT and reported an $89 \%$ complete response rate in 18 head-and-neck cancer patients [10]. In this study, $83.7 \%$ and $84.8 \%$ of the patients in SEQ-IMRT and SIB-IMRT had complete remission.

We used concurrent weekly cisplatin since it has been proven for its efficacy, better compliance and convenience as compared to three-weekly regimens [26]. For adjuvant chemotherapy, cisplatin $80 \mathrm{mg} / \mathrm{m}^{2}$ on Day 1 combined with $5 \mathrm{FU} 1000 \mathrm{mg} / \mathrm{m}^{2} /$ day for five days was delivered monthly to a total of three cycles as the intergroup 0099 protocol [21]. Among 122 patients in our study, 115 patients $(94.3 \%)$ received at least four cycles of concurrent chemotherapy, compared with $78 \%$ in the study of Chan [26], which was higher than a three-week regimen in other studies. Most of our patients (65.6\%) completed three cycles of adjuvant chemotherapy, which was higher than the compliance rate in intergroup 0099 [21] and RTOG 0225 [13] studies, at $55 \%$ and $46.5 \%$, respectively.

Our primary outcome was to assess acute and late toxicities using Common Terminology Criteria for Adverse Events (CTCAE) version 4.03. All patients experienced some degrees of acute toxicities, most of which were of mild severity, grade $1-2$. Weight loss and oral mucositis were the main problems during treatment. No significant difference in acute and late toxicities between two IMRT techniques was observed.

Because different criteria were used for the toxicity evaluation, comparing the toxicity among several published trials might not be appropriate. In RTOG 0225 which used similar toxicity evaluation criteria, acute grade 3 mucositis, defined as confluent pseudomembranous reaction (contiguous patches generally $>1.5 \mathrm{~cm}$ in diameter) in CTCAE version 2.0 and as severe pain or interfere oral intake in CTCAE version 4.03 , was $29.4 \%$ in the RTOG study [13] compared to $13.9 \%$ in our study. There was one patient who experienced acute grade 5 mucositis in both trials, accounting for $4.4 \%$ and $0.8 \%$ in RTOG study and our study, respectively. Grade 3 or more acute mucositis in our study is less than in the others, thanks to the dose volume constraint we put to the oral cavity.

The rate of grade 3 dermatitis, which was defined as confluent moist desquamation $\geq 1.5 \mathrm{~cm}$ diameter and not confined to skin folds or pitting edema in CTCAE version 2.0 and as moist desquamation other than skin folds and creases or bleeding induced by minor trauma or abrasion in CTCAE 4.03, was $13.2 \%$ in the RTOG study [13] compared to $8.2 \%$ in our study.

Late grade 2 xerostomia in the three-month period in our study was $9.5 \%$ compared to $64 \%$ reported by UCSF [5]. At 12 months, late grade 2 xerostomia in our study was only $0.8 \%$ compared with $32 \%$ and $13.5 \%$ in MSKCC [6] and RTOG 0225 [13] studies, respectively. Of note were the different toxicity criteria among these studies.

Regarding significant anatomic changes during RT, including tumor shrinkage and changes in body contour due to weight loss, these factors can affect the dose distribution to the target volume and normal tissue, which can result in inadequate target coverage or increased toxicities. Notably, the proportion of T4 and N3 staging, PTV volume as well as the incidence of weight loss during CCRT was slightly higher in SIBIMRT thus an increased toxicity in SIB-IMRT was possible. However, the current preliminary data did not show the significant difference between both IMRT techniques. Several studies reported the excellent outcomes on disease control and late toxicity of adaptive radiation treatment in NPC patients, especially those with advanced $\mathrm{T}$ and $\mathrm{N}$ stage [27]. Therefore, we anticipate that patients with larger PTV volume treated with SEQ-IMRT would get better results from two treatment-planning with re-CT simulation after half way of treatment.

With a median follow-up time of 16.8 months, locoregional recurrence was found in only one patient and distant metastasis occurred in nine patients in SIB-IMRT. On analysis of tumor response, pattern of failure and survival, there was no significant difference between the two different IMRT techniques, except for the rate of distant metastasis $(p=0.005)$. The higher distant metastasis rate in SIB-IMRT might be explained by higher proportion of T4, N3 and stage IV disease despite insignificant difference between both treatment arms. 
The limitation of this study was that it was nonblinded, as the same physicians were responsible for each subject through the process of treatment planning and follow-up, thus they were not blinded from treatment group, resulting in possible observer bias. Neither was the patients blinded because of different numbers of radiotherapy fractions. Additionally, the diagnostic techniques for staging and re-staging used in this trial was mainly CT scan of the nasopharynx and chest xray instead of MRI of the nasopharynx and CT scan of the chest, respectively, due to technical availability limitations. However, patients whom were of potential benefit from these techniques, such as T4 patients and those with abnormal $\mathrm{x}$-ray, would be strongly considered. Furthermore, as this is a preliminary result we plan to allocate and randomize additional patients in order to reach significant statistical analysis. Long-term follow-up is needed to demonstrate efficacy on disease control and survival as well as late toxicities.

\section{Conclusion}

Preliminary results from this study showed that SEQIMRT has comparable toxicity to SIB-IMRT. Short-term tumor control was promising. However, more patient accrual and longer follow-up are needed.

\section{Abbreviations \\ 3D-CRT: 3D conformal radiation therapy; BED: Biological equivalent dose; CT: Computed tomography; CTCAE: Common terminology criteria for adverse events; MRI: Magnetic resonance imaging; NPC: Nasopharyngeal carcinoma; OS: Overall survival; PET: Positron emission tomography; PTV- LR: Low-risk planning target volume; PTV-HR: High-risk planning target volume; PFS: Progression-free survival; RECIST: Response evaluation criteria in solid tumors; SEQ-IMRT: Sequential intensity modulated radiation therapy; SIB-IMRT: Simultaneous integrated boost intensity modulated radiation therapy; SPSS: Statistical Packages for Social Sciences; WHO: World Health Organisation.}

\section{Competing interests}

The authors declare that they have no competing interests.

\section{Authors' contributions}

AS participated in carried out the study design and data acquisition, performed the statistical analysis and drafted the manuscript. CL conceived of the study, and participated in its design and coordination together with AS and helped to draft and revise the manuscript. All authors participated in radiotherapy planning, treatment, evaluation and follow-up. All authors read and approved the final manuscript.

\section{Acknowledgements}

This research article is made possible through the help and support from significant advisors and industrious colleges. Thank you for Faculty of Medicine, Chulalongkorn University and King Chulalongkorn Memorial Hospital, Thai Red Cross Society for providing the resources and funding for quantitative measurement of plasma EBV DNA level. In addition, we sincerely thank to our family who gave all supports. The product of this research paper would not be possible without all of them.

Received: 12 February 2015 Accepted: 22 July 2015

Published online: 08 August 2015

\section{References}

1. Khuhaprema T, Attasara P, Striplung H, Wiangnon S, Sangrajrang S. Incidence of nasopharyngeal cancer in Thailand. Cancer Thailand 2007. 2009;7:20-2.

2. Nutting C, Dearnaley DP, Webb S. Intensity modulated radiation therapy; a clinical review. Br J Radiol. 2000;73:459-69.

3. Eisbruch A, Ten Haken RK, Kim HM, Marsh LH, Ship JA. Dose, volume, and function relationships in parotid salivary glands following conformal, and intensity-modulated irradiation of head and neck cancer. Int J Radiat Oncol Biol Phys. 1999:45:577-87.

4. Xia P, Fu KK, Wong GW, Akazawa C, Verhey LJ. Comparison of treatment plans involving intensity modulated radiotherapy for nasopharyngeal carcinoma. Int J Radiat Oncol Biol Phys. 2000;48:329-37.

5. Lee N, Xia P, Auivey JM, Sultanem K, Poon I, Akazawa C, et al. Intensitymodulated radiotherapy in the treatment of nasopharyngeal carcinoma. An update of the UCSF experience. Int J Radiat Oncol Biol Phys. 2002;53:12-22.

6. Wolden SL, Chen WC, Pfister DG, Kraus DH, Berry SL, Zelefsky MJ. Intensitymodulated radiation therapy (IMRT) for nasopharyngeal cancer. Update of the Memorial Sloan Kettering experience. Int J Radiat Oncol Biol Phys. 2006;64:57-62.

7. Pow EH, Kwong DL, McMillan AS, Wong MC, Sham JS, Leung LH, et al. Xerostomia and quality of life after Intensity-modulated radiation therapy and conventional radiotherapy for early-stage nasopharyngeal carcinoma. Initial report on a randomized controlled clinical trial. Int J Radiat Oncol Biol Phys. 2006;66:981-91.

8. Butler EB, Teh BS, Grant WH, Uhl BM, Kuppersmith RB, Chiu JK, et al. SMART (simultaneous modulated accelerated radiation therapy) boost: a new accelerated fractionation schedule for the treatment of head and neck cancer with intensity modulated radiotherapy. Int J Radiat Oncol Biol Phys. 1999:45:21-32.

9. Withers HR, Taylor JMG, Macejewski B. The hazard of accelerated tumor clonogen repopulation during radiotherapy. Acta Oncol. 1988;27:131-46.

10. Lertbutsayanukul C, Khorprasert C, Shotelersuk K, Jumpangern C, Sanghangthum T, Oonsiri $S$, et al. Intensity-modulated radiation therapy in head-and-neck cancer, first report in Thailand. J Med Assoc Thai. 2006:89:2068-76

11. Chen SW, Yang SN, Liang JA, Shiau AC, Lin FJ. Comparative dosimetric study of two strategies of intensity-modulated radiotherapy in nasopharyngeal cancer. Med Dosim. 2005;30:219-27.

12. Bucci M, Xia P, Lee N, Fishbein, A. Kramer, V. Weinberg, et al. Intensity modulated radiation therapy for carcinoma of the nasopharynx: an update of the UCSF experience. Int J Radiat Oncol Biol Phys. 2004;60:317-8.

13. Lee N, Harris J, Garden AS, Straube W, Glisson B, Xia P, et al. Intensitymodulated radiation therapy with or without chemotherapy for nasopharyngeal carcinoma: Radiation Therapy Oncology Group Phase II Trial 0225. J Clin Oncol. 2009;27:3684-90.

14. Amosson CM, The BS, Huang E, Uy N, Huang E, Mai WY, et al. Accelerated Fractionation for Head and Neck Cancer Using the SMART (Simultaneous Modulated Accelerated Radiation Therapy) Boost Technique. Int J Radiat Oncol Biol Phys. 2001;51:179-80.

15. Xie CY, WU SX, Jin XC, Yu JY, Wang JH, Li WF, et al. Simultaneous modulated accelerated radiation therapy in the treatment of nasopharyngeal cancer. Zhonghua Yi Xue Za Zhi. 2007;87:2412-5.

16. Wong FC, Ng AW, Lee VH, Lui CM, Yuen KK, Sze WK, et al. Whole-field simultaneous integrated-boost intensity-modulated radiotherapy for patients with nasopharyngeal carcinoma. Int J Radiat Oncol Biol Phys. 2010;76:138-45.

17. Lee SW, Back GM, Yi BY, Choi EK, Ahn SD, Shin SS, et al. Preliminary results of a phase I/II study of simultaneous modulated accelerated radiotherapy for nondisseminated nasopharyngeal carcinoma. Int J Radiat Oncol Biol Phys. 2006;65:152-60.

18. Peponi E, Glanzmann C, Kunz G, Renner C, Tomuschat K, Studer G. Simultaneous integrated boost intensity modulated radiotherapy (SIB-IMRT) in nasopharyngeal cancer. Strahlenther Onkol. 2010;186:135-42.

19. Thompson LD. Update on nasopharyngeal carcinoma. Head Neck Pathol. 2007;1:81-6

20. Songthong A, Chakkabat C, Kannarunimit D, Lertbutsayanukul C. Efficacy of intensity-modulated radiotherapy with concurrent carboplatin in nasopharyngeal carcinoma. Radio Oncol. 2015;49:155-62.

21. Al-Sarraf M, LeBlanc M, Giri PG, Fu KK, Cooper J, Vuong T, et al. Chemoradiotherapy versus radiotherapy in patients with advanced 
nasopharyngeal cancer: phase III randomized intergroup study 0099. J Clin Oncol. 1998;16:1310-7.

22. Joiner MC. Hyperfractionation and accelerated radiotherapy, Basic clinical radiobiology. London: Arnold; 1997. p. 123-31.

23. Denham JW, Walker QJ, Lamb DS, Hamilton CS, O'Brien PC, Spry NA, et al. Trans Tasman Radiation Oncology Group (TROG). Mucosal regeneration during radiotherapy. Radiother Oncol. 1996;41:109-18.

24. Nesrin D, Stephen K, Bahman E, Najeeb M, Nena M, Leonid BL, et al. Assessment of different IMRT boost delivery methods on target coverage and normal-tissue sparing. Int J Radiat Oncol Biol Phys. 2003;57:1480-91.

25. Orlandi E, Palazzi M, Pignoli E, Fallai C, Giostra A, Olmi P. Radiobiological basis and clinical results of the simultaneous integrated boost (SIB) in intensity modulated radiotherapy (IMRT) for head and neck cancer: A review. Crit Rev Oncol Hemat. 2010;73:111-25.

26. Chan AT, Leung SF, Ngan RK, Teo PM, Lau WH, Kwan WH, et al. Overal survival after concurrent cisplatin-radiotherapy compared with radiotherapy alone in locoregionally advanced nasopharyngeal carcinoma. J Natl Cancer Inst. 2005;97:536-9.

27. Nushimura Y, Shibata T, Nakamatsu K, Kanamori S, Koike R, Okubo M, et al. A Two-step Intensity-modulated Radiation Therapy Method for Nasopharyngeal Cancer: The Kinki University Experience. J Clin Oncol. 2010;40:130-8

\section{Submit your next manuscript to BioMed Central and take full advantage of:}

- Convenient online submission

- Thorough peer review

- No space constraints or color figure charges

- Immediate publication on acceptance

- Inclusion in PubMed, CAS, Scopus and Google Scholar

- Research which is freely available for redistribution 